\title{
GEOGRAFÍA DE LA DEUDA PÚBLICA ESPAÑOLA. ALGUNAS CONSIDERACIONES ESPACIALES DESDE LA PERSPECTIVA POLITICA
}

\author{
Juan Carlos Fernández Cela \\ Departamento de Ciencia Política y de la Administración III. Universidad Complutense de Madrid' \\ juanca01@pdi.ucm.es
}

\section{RESUMEN}

El papel predominante que están jugando los mercados financieros internacionales en la crisis económica que sufre los espacios periféricos de la UE, ha puesto de manifiesto la creciente dependencia e interconexión de los flujos de capital extranjero en la actuación política española, y en su papel en la escena internacional. La elaboración de una cartografía financiera permite identificar algunas características de las sociedades que adquieren deuda soberana española y los países desde donde se compra y venden estos productos. Es posible que exista una relación de dependencia entre esta geografía de las finanzas y el margen político de actuación de España en este contexto de crisis.

Palabras clave: geografía financiera, deuda España, centros financieros internacionales, flujos capital, estructura espacial sociedades financieras.

\section{ABSTRACT}

The predominant role international financial markets are playing in the economic crisis faced by the peripheral areas of the EU, have highlighted both the growing dependence and the interconnection of foreign capital flows into the Spanish political action and its role in the international scene. The drafting of some kind of financial mapping helps us identify

Fecha de recepción: enero 2013.

Fecha de aceptación: diciembre 2013.

1 Me gustaría agradecer especialmente a Ricardo Méndez y María Lois sus comentarios, críticas y aportaciones; han sido de gran utilidad para mejorar la calidad de este trabajo. Lo hago también extensivo a los evaluadores anónimos de la AGE, así como a Rocío Chico del Tesoro Público; Alfonso Núñez, del Banco de España; Juan Mascareñas, de la UCM; y por último a Francesca Coloni y Javier Álvarez de la agencia Thomson Reuters por su colaboración en el acceso a la información financiera y apoyo técnico, que ha resultado imprescindible para la realización de este artículo. 
some of the characteristics of the companies which are acquiring Spanish sovereign debt as well as the countries where those products are sold and bought. The existence of a mutual relationship of dependency between this geography of finance and Spain scope for action within this framework of financial crisis may be the cause of the former phenomenon.

Key words: financial geography, Spain debt, international financial centers, equity flows, spatial structure finance companies.

\section{INTRODUCCIÓN}

La crisis económica actual, que está afectando a una parte importante de los países de la UE, tiene un impacto que supera el propio ámbito económico para poner en tela de juicio la viabilidad actual del propio modelo de proyecto europeo. A pesar de la gran cantidad de estudios e informes que están saliendo sobre esta cuestión, se percibe una cierta sensación de desconcierto generalizado ante esta situación, que está desbordando todos los marcos conocidos en los últimos años de una cohesión en el seno de la UE que hasta hace muy poco se percibía como sólida.

En el caso concreto de España la depresión económica está teniendo una especial virulencia, en la medida en que se está poniendo en cuestión la solvencia financiera del sector público y privado. Este período de recesión provoca numerosas tensiones en los mercados financieros internacionales como consecuencia de la inestabilidad y de la imposibilidad de conocer, en este momento, cuándo y cómo se va a producir de nuevo una nueva fase de crecimiento que reduzca el elevado déficit y sea capaz de generar empleo y actividad.

¿Qué puede aportar la geografía al conocimiento de la crisis económica? Entre todas las posibilidades hay una perspectiva que no ha sido ampliamente tratada en España y que quizás sea importante considerar, especialmente desde una visión de análisis territorial a medio o largo plazo. Es la que hace referencia al análisis espacial de los movimientos internacionales de capital, con especial énfasis en aquellos ligados a la inversión en deuda pública española, que parece encontrarse en el epicentro de la propia crisis.

El principio de partida se basa en que la estructura espacial de las relaciones financieras internacionales, es un elemento que puede ayudar a conocer mejor las características de la crisis económica actual. Existe una creciente interdependencia entre los flujos de capital, inversiones y los mecanismos de toma de decisiones en materia de política financiera. Los mercados de capital tienen una notable capacidad de influencia en el margen de maniobra político de los Estados, su solvencia financiera y en definitiva la capacidad de implementar un determinado modelo de Estado.

En este contexto las relaciones espaciales adquieren una importancia relevante, en la medida en que la morfología de las redes de origen y destino del capital pueden determinar una mejor o peor capacidad de financiación de los Estados. La posibilidad de poder cartografiar los movimientos internacionales de capital puede ser una línea útil de investigación para la geografía, en la medida en que pueda aportar algunos datos relevantes sobre la procedencia de las inversiones y la influencia de la localización espacial en la estructura organizativa de las «multinacionales» financieras que realizan operaciones a escala global pero desde una lógica espacial diferenciada. 


\section{OBJETIVOS Y METODOLOGÍA}

A la hora de abordar el método más adecuado para analizar desde una perspectiva geográfica el flujo de inversiones financieras en la deuda, es necesario tener en cuenta las dificultades que existen de obtener una información precisa sobre este aspecto. El primer obstáculo está ligado a la complejidad que existe en trasvasar información financiera al ámbito de análisis espacial. Los indicadores, variables e información en general que se utilizan habitualmente en el mercado de valores no tiene una finalidad estadística, por lo que es en ocasiones complicado conocer con exactitud qué es exactamente lo que representa un determinado indicador. Para investigar más a fondo la relación entre los actores financieros y su papel en la configuración del espacio de las finanzas globales, se requiere un tipo diferente de información. Tal y como se pone de manifiesto en algún estudio reciente, la falta de datos relacionales sigue siendo un gran obstáculo para las aplicaciones prácticas de estas nuevas perspectivas teóricas (Seo, 2011).

Hay una segunda dificultad vinculada al elevado componente inmaterial que tiene este tipo de flujos de capital. Es más factible sistematizar los movimientos origen-destino de personas o bienes. Sin embargo, el flujo de capital es más complejo de analizar debido entre otras cuestiones a que se trata de un mercado intangible, con unas normas de funcionamiento complejas, una escala global y de carácter continuo, por lo que es posible movilizar ingentes cantidades de capital de un punto a otro de forma muy rápida y en cualquier momento del año, durante las 24 horas del día.

A pesar de ello, las tendencias de investigación que están comenzando a surgir en otros países tienen una dinámica cuantitativa y cualitativa diferente. Algunos economistas han comenzando a analizar los flujos financieros de inversión y sus efectos espaciales en la crisis financiera. Se habla ya de una «geografía de la transferencia de riqueza». Tomando como punto de partida el comportamiento de algunos índices financieros de transferencia de capital, se intenta conocer la movilidad espacial del capital financiero en activos inmobiliarios y sus efectos sobre los espacios de origen y destino de las mismas (Gourinchas, Rey y Truempler, 2011). Se trata principalmente de estudios económicos que concentran su interés en el impacto que tiene en los mercados financieros internacionales. No son por tanto estudios espaciales, aunque sí consideran el territorio como una variable estratégica. Más cercanos a la geografía son algunos estudios donde se realiza un análisis de las implicaciones geopolíticas de la geografía del dinero a través de la especialización de los centros financieros internacionales en determinados productos de inversión (French, Leyshon y Thrift, 2009).

Por lo tanto, un acercamiento mínimamente riguroso a este problema requiere un esfuerzo multidisciplinar, especialmente entre geógrafos y economistas, que excede con mucho el alcance de este artículo. Por ello, quizás sea más eficaz delimitar un aspecto concreto del mismo que está teniendo en la actualidad un impacto espacial, económico, político y social muy destacado. El objetivo de este trabajo se centra en analizar la estructura espacial de las sociedades financieras extranjeras que invierten en el mercado español de deuda pública.

Al igual que ocurre en otros sectores económicos, la organización de las empresas financieras internacionales tiene una lógica territorial muy característica. Como podrá 
observarse en los datos aportados, los inversores extranjeros en deuda española, catalogados por el Tesoro Público bajo la denominación genérica de «tenedores no residentes», conforman un conglomerado de empresas que tienen una estructura organizativa compleja, donde la dimensión espacial juega un papel central en su estrategia de mercado global.

Es importante señalar que desde el punto de vista del análisis territorial hay una diferencia importante entre el concepto de inversor extranjero y tenedor no residente. La complejidad de la actividad financiera, ha provocado que sociedades de origen español puedan adquirir deuda desde filiales localizadas en otros países. Esto supone por tanto que el inversor tendría un origen nacional pero estaría catalogado como tenedor no residente. Por el contrario, sociedades de origen foráneo pueden adquirir deuda desde sus filiales localizadas en España. En este caso, más habitual e importante que el anterior, serían inversores extranjeros, aunque la adquisición de la misma no se realiza en el exterior. Los datos geográficos aquí analizados hacen referencia la localización concreta de las sociedades de inversión. Por ejemplo, si un inversor en deuda española es la filial localizada en Irlanda de una sociedad de origen francés, a efectos del cómputo espacial el capital declarado se asigna a Irlanda y no a Francia.

Junto a la escala espacial, ha sido necesario determinar con claridad la unidad de análisis más adecuada. En este caso se ha tomado como referencia las Obligaciones del Estado con vencimiento a 10 años. Es un tipo de activo que se utiliza habitualmente como punto de referencia a la hora de identificar la confianza del mercado en la deuda de un determinado país, como es el caso de la prima de riesgo. Se trata pues de emisiones en el mercado secundario de obligaciones, títulos que declaran tener en sus carteras las sociedades de inversión en agosto de 2012.

Resulta cuanto menos significativa la ausencia en las estadísticas oficiales de determinada información financiera. Ni el Tesoro Público ni el Banco de España, ofrecen en sus publicaciones datos detallados sobre los tenedores no residentes que adquieren deuda pública. La escasa información ofrecida en las estadísticas oficiales de Tesoro Público tiene un curioso nivel de desagregación espacial. Por países en tres casos (Francia, Alemania e Italia); amplias zonas geográficas en otros (América; África, Asia y «otros»); económica (Benelux) o delimitaciones poco definidas («Resto de Europa»). Como es evidente para cualquier geógrafo, esto complica el análisis espacial en la medida en que no es posible obtener una mínima información homogénea desde el punto de vista territorial.

Al no ser posible obtener la desagregación espacial adecuada en los registros públicos, se ha optado por analizar diversos datos procedentes de la agencia financiera Thomson Reuters, que dispone de información actualizada clave para poder avanzar y conocer algunas características de los tenedores no residentes. El acceso a sus sistemas informáticos ha permitido obtener información territorial muy relevante sobre, entre otras cuestiones, el valor nominal de la deuda declarada por las sociedades financieras, y especialmente la localización exacta de las distintas filiales que estas empresas tienen por todo el mundo. A través de la agencia se ha podido acceder a la relación de las sociedades de inversión que han declarado tener títulos de deuda pública en agosto de 2012, última fecha disponible a la hora de redactar este trabajo. El cruce de los bancos de datos disponibles en la agencia ha permitido disponer de una relación de establecimientos desagregada por países que declaran poseer su tenencia. 
Las emisiones seleccionadas corresponden exclusivamente a las realizadas en el mercado secundario de compraventa de valores, y se han localizado a través de sus códigos ISIN ${ }^{2}$. La información obtenida afecta exclusivamente a aquellas sociedades que han declarado poseer estos productos en sus carteras. Se estima que la información analizada representa alrededor de un $4 \%$ del valor nominal total de las emisiones registradas. Se trata de una reducida proporción del mercado global de deuda, pero es la única existente y se concentra exclusivamente en el mercado secundario, el más importante para el análisis de la movilidad espacial del capital. La información utilizada en este trabajo debe considerarse como una primera aproximación, que se podría mejorar en la medida en que puedan aparecer nuevas fuentes de información estadística, especialmente de origen público.

\section{MOVILIDAD DEL CAPITAL Y SU IMPACTO ESPACIAL}

Al igual que existe un espacio económico tangible basado en la movilidad de bienes y servicios, podría hablarse de una geografía financiera donde se determina los flujos de inversiones con puntos de origen y destino del capital. En su interior hay un elevado nivel de movilidad, permitiendo en cuestión de segundos numerosas transacciones financieras de un punto a otro del mundo sin apenas trabas o tasas. La introducción de nuevas tecnologías en los mercados ha acelerado la volatilidad del capital, multiplicando de forma exponencial en caso de crisis la probabilidad de riesgo y contagio en cada espacio económico y político.

La existencia de puntos neurálgicos de poder financiero desde los que se determina dónde, cuánto y cómo se invierte en este u otro espacio geográfico constituyen nodos centrales de una red financiera que, como premisa de partida, es clave a la hora de poder comprender algunos de los fenómenos que estamos viviendo en la crisis actual. Existen por tanto determinados espacios estratégicos «core» localizados principalmente en los grandes centros financieros internacionales, donde se desarrollan innovaciones que combina por un lado la tecnología de la comunicación, que mejora sustancialmente la calidad y velocidad en la transmisión de datos entre diferentes espacios económicos, y la tecnología de la información, vinculada a la creación de herramientas para la toma de decisiones sobre los productos y regiones donde se invierte, que garantiza la movilidad global del capital.

A todo esto habría que sumar un complejo entramado de plataformas financieras -entre las que destaca FOREX, el mayor mercado mundial especializado en la inversión en moneda-, diseñadas para obtener una información financiera en tiempo real que permita movilizar las inversiones de un punto a otro con el objetivo de obtener el mayor beneficio.

Desde una óptica territorial, los flujos financieros internacionales están jugando un papel determinante en el sistema financiero de los países más afectados por la crisis, especialmente en lo que respecta a la capacidad de financiación del déficit público y de la deuda en los mercados internacionales. Algunas líneas recientes de investigación se encuentran ligadas a la transferencia masiva de riqueza entre espacios centrales y periféricos. El mapa de riesgo de determinados países se ha modificado en función de las inversiones en determinados productos financieros complejos y su nivel de exposición a un riesgo creciente (Gourinchas, Rey y Truempler, 2011).

2 International Securities Identification Number, un código internacional que permite identificar valores mobiliarios. Se usa como referencia habitual en los mercados financieros internacionales para la compraventa de títulos. 
Una explicación al menos parcial de este elemento podría estar ligada a la existencia de un desplazamiento del eje de gravedad, el denominado «heat map», desde las plazas tradicionales del Atlántico, que están perdiendo importancia a favor de los centros financieros del Pacífico. Recientes trabajos consideran que comienza a dibujarse desde 2001 un nuevo mapa económico global a partir de los cambios producidos en la industria financiera. Esta «reestructuración productiva» del sector financiero mundial ha supuesto aproximadamente la disminución de una quinta parte del empleo existente en 2007, reforzado por el creciente proceso de desregulación de los mercados globales (Capelle-Blancard y Tadjeddine, 2010).

En efecto, la mayor complejidad y la dimensión internacional de determinados productos financieros generan cambios territoriales ligados a una nueva estructura bancaria global. La jerarquía financiera internacional ha sufrido una transformación destacada como consecuencia de la pérdida de influencia de algunos centros financieros tradicionales, especialmente Londres y Nueva York, que coincide con el auge de la los mercados de valores de diversos centros situados en economías emergentes que les eclipsan como actores principales (Alessandrini, Fratianni y Zazzaro, 2009).

El impacto geográfico de este proceso en la estructura global es evidente. Los datos relativos al Índice Global de Centros Financieros 2012 ponen de manifiesto que la lógica de los flujos monetarios va más allá de un simple desplazamiento hacia el Pacífico. Solo dos ciudades europeas, Londres $\left(1^{\mathrm{a}}\right)$ y Zúrich $\left(6^{\circ}\right)$ se sitúan entre las diez mayores plazas financieras mundiales. Sin embargo destaca en esta relación de centros privilegiados la presencia cinco ciudades asiáticas, Hong Kong, Singapur, Tokio, Shanghái y Seúl (The Z/Yen Group, 2012). Incluso dentro de los propios espacios emergentes el impacto de la crisis financiera ha sido desigual en sus distintos centros; Abu Dabi, Dubái y Riad por un lado, y Manama, Pekín, Shanghái y Kuala Lumpur por otro han tenido salidas diferentes de la crisis financiera (Derudder, Michael y Taylor, 2011).

El análisis desde una perspectiva crítica pone de manifiesto dos aspectos a considerar. El primero de ellos se basa en que la génesis de la crisis financiera viene derivada de las contradicciones que genera el propio modelo de acumulación de capital. Wallerstein habla de una crisis estructural del sistema-mundo provocada, entre otras premisas, porque el plusvalor obtenido se ha diseminado desde los espacios centrales de acumulación hacia las nuevas economías emergentes ya que «el aumento significativo de los estándares de vida de segmentos de la población de los denominados países BRIC ha venido a agravar, de hecho, los problemas de acumulación de los capitalistas, al diseminar la plusvalía y, con ello, reducir el monto disponible para la delgada capa superior de la población de las sociedades mundiales» (Wallerstein, 2012).

Agnew señala que bajo la complejidad en la gestión y manejo de instrumentos financieros se produce una cierta desnaturalización política del discurso económico, que entra dentro de lo técnico para dejar fuera la clave política y social. Las tecnologías financieras generan un cierto hipnotismo que limitan aparentemente el papel humano en el uso y gestión de la movilidad de capital. Se trata de «mercados financieros que operan como si estuvieran fuera del control humano» (Agnew, 2012).

Precisamente este es uno de los principales argumentos del discurso neoliberal sobre el carácter «inevitable» de realizar una reforma drástica del Estado de bienestar, recortando derechos sociales y privatizando gradualmente servicios públicos, especialmente educación 
y sanidad. La consecuencia intelectual más poderosa ha sido la eficaz despolitización de los debates de fines económicos debido a su carácter esencialmente tecnocrático. La complejidad de la estructura, contenidos y el propio lenguaje financiero constituye un arma poderosa para desnaturalizar el contenido político del debate económico. El análisis se deja en manos de expertos tecnócratas que utilizan así numerosos argumentos como hábil justificación de las grandes decisiones, especialmente los recortes de la políticas sociales, que según su propia lógica no se pueden obviar.

Debemos por tanto realizar un cierto esfuerzo intelectual a la hora de separar el análisis de la información financiera y la toma de decisiones política que no está, en modo alguno y en contra de lo que muchos intereses quieren hacer pensar, implícita en la toma de medidas que a la postre suponen la implantación de un modelo neoliberal y el desmantelamiento progresivo de unos derechos sociales que ha garantizado durante décadas una estabilidad económica, social y política.

Bajo determinadas circunstancias, especialmente en un contexto de crisis económica como la que vivimos en la actualidad, la capacidad de diversos Estados de influir en las inversiones dirigidas a uno u otro país determinado puede suponer un elemento de presión política para modificar las relaciones económicas y sociales básicas del Estado que recibe las ayudas. El conflicto de este teatro de operaciones financiero se traslada de un espacio físico a uno virtual de dimensión global que se encuentra congestionado por la cantidad de actores que intervienen en él. Es un lugar confuso debido a la falta de transparencia en numerosas operaciones financieras, donde la circulación del capital en paraísos fiscales hace difícil establecer su destino final. Se trata también de un territorio de disputa geopolítica, en la medida en que las inversiones financieras pueden ejercer presiones de carácter político. Es finalmente un espacio altamente interconectado y restrictivo, donde las burocracias administrativas que tienen para hacer frente a situaciones de crisis son cada vez más obsoletas, ya que las condiciones han cambiado sustancialmente y exceden su capacidad de control (Puig Soler, 2011).

\section{DISTRIBUCIÓN ESPACIAL DE LOS TENEDORES DE OBLIGACIONES. HACIA UNA CARTO- GRAFÍA DE LOS FLUJOS FINANCIEROS}

Durante el proceso de análisis de la información obtenida se puede identificar la existencia de dos grandes ámbitos o niveles claramente diferenciados que determinan el origen espacial de los inversores financieros internacionales.

El primero corresponde al conjunto de sociedades de inversión en instrumentos de deuda. Se trata de un conjunto de instituciones financieras, muchas de ellas en forma de sociedades anónimas, que gestiona las carteras de inversión colectiva para la compra directa de Deuda pública o la compraventa de títulos en el mercado secundario. Son los que intervienen de forma directa en la adquisición de títulos en los mercados financieros. Aunque en ocasiones pueden disponer de capital propio, la mayor parte procede de inversores externos.

Se trata de un tipo de empresa altamente especializada que dispone de personal con un elevado nivel de cualificación profesional. Están en numerosos casos ligados a grandes bancos (Santander Assets, BBVA Asessts, BNP Paribas Investment, etc.) y funcionan en ocasiones como enlaces que vinculan la actividad bancaria comercial tradicional con la 
inversión financiera en mercados altamente especulativos de derivados y futuros, donde las plusvalías son sustanciales pero también el riesgo que genera el uso de algunas estrategias de inversión, especialmente aquellas vinculadas a técnicas de apalancamiento. Esta unión de facto entre banca comercial y financiera, que permite invertir enormes cantidades de dinero en productos de alto riesgo, es un aspecto clave del problema. Algunos expertos señalan que la derogación durante la administración Clinton de la Ley Glass-Steagall, vigente desde el New Deal y que impedía que la banca comercial tradicional, ligada al mercado de depósitos invirtiera en el mercado de valores, está en el origen de la crisis.

Desde el punto de vista espacial, son sociedades que en numerosas ocasiones tienen una estructura organizativa compleja con un elevado nivel de descentralización organizativa y deslocalización de su actividad en centros financieros de rango global. Los países y ciudades de destino suelen coincidir con los centros financieros mundiales, a través de una estrategia de ampliación y diversificación del mercado. El régimen fiscal, la facilidad para movilizar en cortos períodos y sin grandes obstáculos una ingente cantidad de capital, o el emplazamiento en centros financieros estratégicos son tan solo algunas de las pautas de localización vinculadas a este tipo de actividades.

Aunque la relación interna de cada una de las filiales puede variar, en general el nivel de autonomía no es muy elevado. Existe por tanto una fuerte dependencia con respecto a las decisiones estratégicas tomadas en la sede central, localizada generalmente en los grandes centros financieros mundiales y que ejerce un rígido control piramidal con respecto a las diferentes filiales que hay otros países.

Para la elaboración de este trabajo se ha mantenido algunas conversaciones informales no grabadas con responsables de algunas de las grandes sociedades de inversión mundiales. La mayor parte de ellos coinciden en que el margen de maniobra que tiene las filiales localizadas en España es reducido. Se ha llegado incluso a afirmar que en el mercado español de deuda pública, las verdaderas decisiones no se toman desde Madrid, sino en la City de Londres, con independencia del punto de origen donde se efectúa la orden de compra, o de la localización de las diferentes sociedades, propietarios legales de los valores. Un argumento que apoya esta hipótesis es que la City es el lugar habitual donde los máximos dirigentes españoles explican su política económica a los inversores extranjeros.

Es posible que en algunos casos la decisión final esté vinculada a otros factores diferentes de localización. Una estrategia comercial con perspectiva geoestratégica, o ventajas fiscales de filiales en Estados con estructuras impositivas diferente son algunos que conviene tener en cuenta.

Podría por tanto hablarse de complejos conglomerados mercantiles que operan en el mercado financiero internacional a través de diversas sociedades. Comparten un elevado nivel de descentralización espacial y organizativa de sus filiales, localizadas en numerosas ocasiones en los grandes centros financieros mundiales, especialmente en EE.UU., Reino Unido, Francia, Alemania, Taiwán o Japón. La estrategia de deslocalización permite así abarcar un mercado financiero de mayor dimensión a través de una estructura empresarial más o menos diversificada.

La información disponible analizar con bastante detalle la localización espacial de las sociedades de inversión. Lo primero que se observa es la existencia de un acusado proceso de concentración de las principales empresas financieras en un reducido número de Estados. Este hecho es similar al que ocurre en el resto de los países desarrollados, donde las grandes 
compañías inversoras mundiales se localizan en pocos países. Si se analiza la trayectoria que existe en la deuda declarada entre 2006 y 2012 se advierte que únicamente se han adquirido Obligaciones desde un total de veinticinco Estados diferentes, que año tras año se repiten con regularidad (Mapa 1).

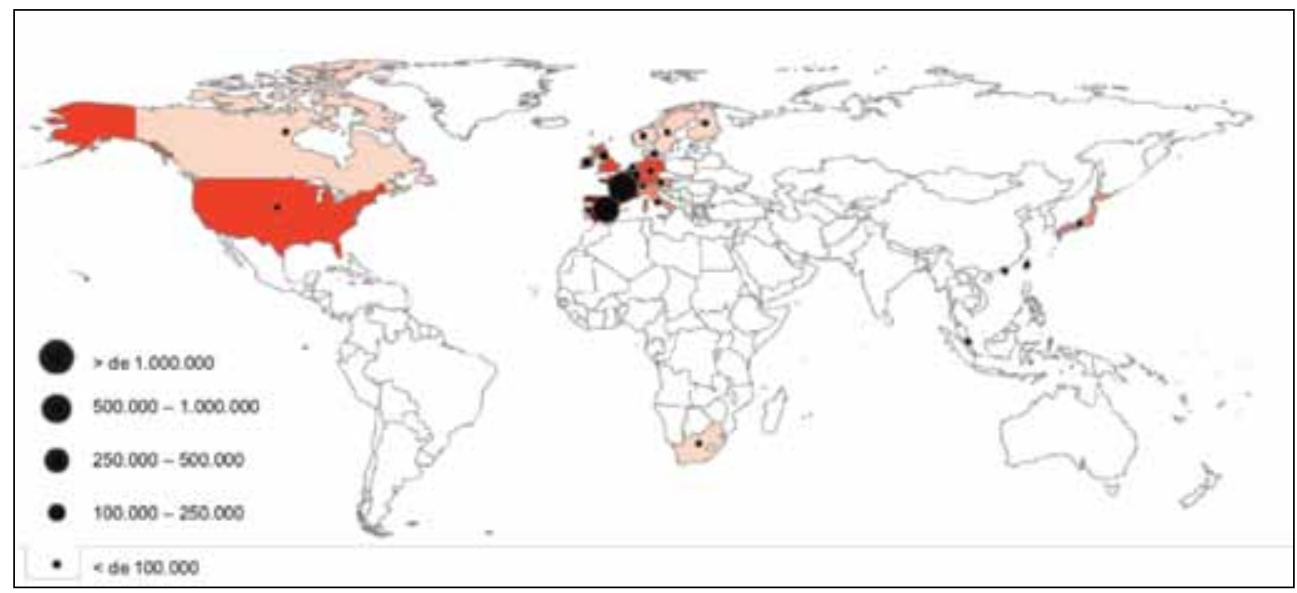

Fuente: Thomson Reuters al 14/08/2012 y elaboración propia.

Desde la óptica de las relaciones financieras internacionales, a pesar de la diversa procedencia del capital, existe una concentración del flujo de inversión en un reducido número de países. Si excluimos las procedentes dentro del propio territorio español, la mayor parte de las sociedades de inversión se localizan en Estados de la UE. En términos generales, la presencia de mercantiles en el espacio americano, asiático y no digamos ya el africano tiene una presencia poco relevante.

El 95,75\% del valor nominal de los títulos analizados ha sido adquirido desde Europa, y el 4,24\% restante se divide a partes iguales entre Asia (2,05\%) y América $(2,19 \%)$. La presencia de sociedades procedentes de África tiene una presencia meramente testimonial $(0,01 \%)$. Parece evidente que el capital de procedencia asiática o americana no opera directamente en el mercado español, sino que lo hace a través de empresas especializadas localizadas en el interior del espacio europeo.

Existen por tanto determinadas plazas financieras mundiales que son críticas a la hora de marcar el ritmo y la dimensión de los flujos de capital que se dirigen a España. Las condiciones y el funcionamiento de las mismas pueden influir tanto en la capacidad de financiación española en el exterior, como en las relaciones políticas que se establece entre España y los países inversores.

Sin embargo, si se observa con mayor detalle la estructura espacial de estos conglomerados, se puede observar como la presencia de Asia y América es mayor de lo esperado. La estructura organizativa de las sociedades financieras más importantes tiene una lógica glo- 
bal, que permite realizar inversiones a través de sus filiales localizadas en otros continentes. Así, no es extraño encontrar empresas de origen estadounidense o japonés que invierten en España a través de filiales localizadas en el espacio europeo e incluso asiático. La presencia de algunos países como Irlanda y Luxemburgo en los puestos altos de inversión se encuentra vinculada a este proceso general de deslocalización. Se trata de un mercado financiero de referencia debido a las ventajas fiscales y estratégicas que tiene la instalación de sociedades. Existen numerosos ejemplos de este proceso, donde la utilización del espacio como elemento estratégico es una práctica habitual. La conexión América-Asia-Europa se pone de manifiesto, por ejemplo, en algunas inversiones de sociedades como Pioneer Investment Management, que proceden de Irlanda pero la matriz es originaria de EE.UU.

Las pautas de localización de las sociedades financieras que operan a escala internacional son sofisticadas. No es posible por el momento realizar un análisis sistemático porque se carece de datos adecuados, pero sí aparecen algunos elementos que pueden ofrecer alguna información al respecto. Parece un hecho factible que el espacio económico es utilizado por estas empresas como elemento de estrategia global. La apertura de numerosas filiales por parte de las sociedades más importantes en distintos puntos es un elemento importante en su mecanismo de toma de decisiones. Existe una presencia casi obligada de las mercantiles más importantes en las plazas financieras de rango global (especialmente Londres, Hong Kong y Nueva York), que se combina con la localización en otras ciudades de rango menor.

Un ejemplo significativo de este tipo de estructura societaria es BNP Paribas. Se trata de un importante banco europeo y comprador habitual en el mercado español. Cuando se analiza con cierto detalle su estrategia en la adquisición de obligaciones podemos ver que las inversiones se realizan a través de al menos ocho delegaciones diferentes localizadas en siete países, aunque no se descarta que este número pudiera ser en realidad superior (Tabla 1).

Tabla 1

ESTRUCTURA ORGANIZATIVA DE BNP PARIBAS. INVERSIÓN DECLARADA DE SUS SOCIEDADES EN OBLIGACIONES (AGOSTO DE 2012)

Valor nominal (en miles de US\$)

BNP Paribas Asset Management SAS (Francia) 782.697

BNP Paribas Luxembourg SA

BNP Paribas Investment Partners London (Reino Unido)

BNP Paribas Investment Partners (Bélgica)

BNP Paribas Investment Partners Japan Ltd

BNP Paribas Investment Partners España, SA, SGIIC

BNP Paribas Investment Partners Netherlands NV

BNP Paribas Asset Management United Kingdom Ltd

Fuente: Thomson Reuters al 21/8/2012 y elaboración propia.

La sede social de la empresa continua estando en Francia, su lugar de origen y el lugar donde declara poseer mayor número de títulos, por encima incluso de su filial española. Junto a ambos países, BNP ha adquirido deuda desde Luxemburgo, Bélgica, Japón, Holanda 
y Reino Unido. La diversificación espacial en la gestión de activos es por tanto elevada. Se desconoce cómo es el funcionamiento interno de este proceso. No se sabe mucho sobre el mecanismo de toma de decisiones que explica por qué se emiten las órdenes de compra desde uno u otro lugar.

El caso de BlackRock es también bastante significativo. De origen estadounidense, ha adquirido en el mercado obligaciones deuda a través de seis filiales localizadas en cinco países: Estados Unidos, Alemania, España, Reino Unido y Japón. Algo parecido ocurre con el caso de la británica HSBC con sus delegaciones en Francia y Hong Kong o la estadounidense JP Morgan por medio de sus secciones en Alemania, Reino Unido, Japón, Taiwán y Hong Kong (Gráfico 1).

En estos casos, la denominación de las sociedades permite identificar con facilidad aquellas que pertenecen a un mismo holding. No ocurre lo mismo cuando los conglomerados societarios son más complejos y por tanto es difícil reconstruir el nivel de concentración de la actividad financiera.

Habría sido tentador intentar recalcular los datos de inversión agrupando como una única sociedad todas las filiales de cada grupo; es muy probable que bajo esta nueva perspectiva se incrementara de forma considerable el nivel de concentración del capital en muy pocas sociedades. Este aspecto es importante, ya que en la práctica podrían controlar de facto la evolución de las inversiones realizadas en el mercado español, con las consiguientes repercusiones políticas y estratégicas que tiene. A pesar de las interesantes posibilidades de análisis que ofrece, no se ha realizado este paso porque en la actualidad no se dispone de información fiable sobre la estructura organizativa de estas empresas y los datos solo podrían recalcularse de forma parcial.

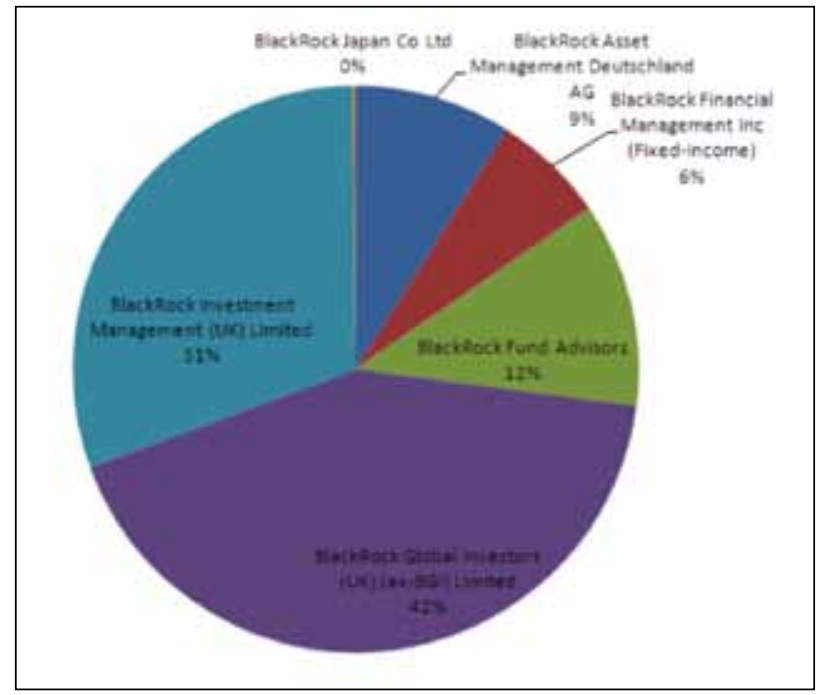

Fuente: Thomson Reuters al 21/8/2012 y elaboración propia. 
Incluso si se prescinde de ello, se puede al menos intuir la existencia de un reducido número de sociedades que en la práctica controlan una parte importante de la deuda española. La Tabla 2 pone de manifiesto la existencia de un elevado nivel de concentración de los tenedores de obligaciones en un selecto grupo de sociedades, con el consiguiente riesgo de cartelización que conlleva este fenómeno. Solo 11 de las 328 sociedades con un identificador diferente absorben más de la mitad $-51,18 \%$ - del valor de todas las obligaciones declaradas. La concentración financiera y la jerarquización de las inversiones es por tanto muy elevada y podría serlo aún más ya que, como hemos visto anteriormente, numerosas empresas que operan en el mercado con diferentes identificadores son en realidad filiales de un gran holding de rango mundial, y por lo tanto se acentúa el nivel de concentración del capital financiero en pocas manos.

Junto a las sociedades, el segundo colectivo clave pertenece a los propios inversores, que son los verdaderos propietarios de los títulos de deuda. Se trata en muchos casos de grupos de distinto tamaño agrupados en fondos de pensiones o de inversión, sociedades de garantía recíproca, entidades de crédito o seguros, empresas no financieras, administraciones públicas, e incluso personas físicas. Un caso particular es el de los fondos soberanos, entidades estatales que gestionan una ingente cantidad de activos en los mercados internacionales. Los fondos proceden en muchos casos aunque no exclusivamente de las plusvalías obtenidas por el incremento de los precios de materias primas como el petróleo; de ahí que muchos de ellos proceden de países exportadores de crudo y gas.

Los datos analizados no permiten tener una información relevante sobre este segundo grupo. Se desconoce el número de inversores, su lugar de procedencia, el tipo de instrumentos financieros que utiliza, etc. Existen algunas referencias imprecisas declaradas por diversas sociedades de inversión sobre la denominación de varias Sociedades de Garantía Recíproca (SGR). Sin embargo, no ofrece datos adicionales sobre su composición interna, localización y naturaleza de la inversión, por lo que no se ha podido utilizar información alguna para la realización de este trabajo.

Desde el punto de vista de análisis espacial es importante diferenciar la procedencia de ambos colectivos, ya que el proceso de globalización financiera permite una mayor integración de los mercados de capital, de tal forma que una sociedad de inversión gestione capitales procedentes de diferentes países y espacios económicos.

En consecuencia, el lugar de origen de los inversores puede ser diferente de la localización de la sociedad que declara poseer sus títulos. El mecanismo de toma de decisiones y gestión de la inversión pueden ser complejo y sofisticado desde el punto de vista espacial. Por ejemplo, una orden emitida desde la City de Londres puede hacer que un fondo soberano taiwanés pueda contratar los servicios de una sociedad de inversión luxemburguesa, a través de su filial localizada en Francia, para adquirir bonos españoles en una subasta determinada. La ruta financiera del ejemplo recorre cinco países diferentes desde su punto de origen a su lugar de destino.

A la hora de determinar la procedencia de la inversión financiera exterior, la información que se está analizando es la correspondiente a la localización de la sociedad mercantil que declara la tenencia de bonos, en el ejemplo sería Francia, y no el propietario real de los mismos, en este caso el fondo soberano de Taiwán. Como es evidente, cambia por completo el resultado del análisis espacial si se toma como referencia la localización de las sedes sociales 
de las sociedades de inversión, o si por el contrario consideramos como punto de referencia espacial la localización de los inversores que contratan los servicios de estas sociedades. Sería muy interesante aunque complejo realizar una «hoja de ruta» financiera que permitiera identificar los flujos origen-destino del capital financiero y los puntos intermedios por donde circula. Quizás podría ofrecer resultados reveladores.

Tabla 2

VALOR NOMINAL DE LAS OBLIGACIONES DECLARADAS POR LAS MAYORES SOCIEDADES DE INVERSIÓN (AGOSTO DE 2012)

\begin{tabular}{lrr} 
& Valor nominal (en miles US\$) & \% \\
\cline { 2 - 3 } & Santander Asset Management SA, SGIIC & 7,25 \\
BBVA Asset Management, SA, SGIIC & 896.282 & 7,20 \\
BNP Paribas Asset Management SAS & 782.697 & 6,29 \\
AXA Investment Managers Paris & 712.128 & 5,72 \\
Amundi & 575.850 & 4,63 \\
Invercaixa Gestión, SGIIC, SAU & 507.497 & 4,08 \\
Allianz Global Investors France SA & 406.713 & 3,27 \\
CM-CIC Asset Management & 346.439 & 2,78 \\
Natixis Assurances & 342.690 & 2,75 \\
MMA Finance & 325.617 & 2,62 \\
CNP Assurances (Caisse Nationale de Prévoyance) & 301.309 & 2,42 \\
Total grandes sociedades & $\mathbf{6 . 3 7 2 . 4 0 6}$ & $\mathbf{5 1 , 1 8}$ \\
\hline Resto de sociedades & 6.078 .253 & 48,82 \\
\hline Total general & $\mathbf{1 2 . 4 5 0 . 6 5 9}$ & $\mathbf{1 0 0 , 0 0}$ \\
\hline
\end{tabular}

Fuente: Thomson Reuters al 14/08/2012 y elaboración propia.

El problema más importante es la imposibilidad de conocer con rigor la procedencia final real de los fondos. La discreción de las sociedades de inversión en este punto es absoluta, comprensible por otro lado dado el carácter delicado de este tipo de información. Aventurar que una sociedad radicada en Estados Unidos gestiona mayoritariamente capital procedente de este país u otros de su entorno entra dentro de lo que podría considerarse como probable, pero se trata en último caso de especulaciones sin una base real, y por tanto no se puede identificar de manera automática el lugar de procedencia de la sociedad con su espacio geográfico de influencia.

\section{IV.1. La concentración de inversores en Europa}

El origen de las inversiones se realiza desde un selecto grupo de países, a partir de los cuales operan las sociedades de inversión que adquieren las obligaciones en el mercado secundario. La mayor parte de las mismas proceden de Estados pertenecientes a la zona euro (España, Francia, Alemania, Italia, Austria, Holanda, Finlandia, Luxemburgo, Irlanda, Bélgica, Portugal y Mónaco). A estos habría que añadir el caso del Reino Unido, Dinamarca 
y Suecia como Estados de la UE sin moneda única, y por último Estados que no pertenecen a la UE (Noruega y Suiza). Por tanto, diecisiete de los veinticinco países de adquisición de deuda se localizan en Europa. Este elemento da idea por un lado del marcado carácter «regional» que tiene la posición financiera española en el contexto internacional, y en segundo lugar la baja participación de las economías emergentes en el mercado de Deuda pública (Mapas 2 y 3 ).

La mayor inversión mundial en obligaciones se realiza desde Francia. Sus sociedades gestionan una deuda emitida por valor de 4.969 millones de dólares, un 39,92\% del valor total, por encima incluso de los títulos adquiridos en España, que para el mismo período alcanza los 4.304 millones y representa un 34,57\%. Siete de cada diez dólares declarados en obligaciones proceden por tanto de sociedades francesas y españolas.

Esta macrocefalia financiera se pone de manifiesto cuando se observa el volumen de adquisición de deuda por parte de otros países. El siguiente inversor en cuanto al valor nominal declarado corresponde a empresas localizadas en Reino Unido, con 498 millones, una cifra muy inferior a la francesa. Lo mismo ocurre con Alemania, con obligaciones declaradas por valor de 318 millones es el quinto inversor europeo. Sus sociedades gestionan una cantidad significativamente pequeña en comparación con la francesa. No obstante, no parece que exista una correlación directa entre la posición económica de un Estado y el volumen de deuda que se adquiere desde él. La estrategia de diversificación espacial que caracteriza a las sociedades financieras desvincula ambos elementos, ya que Allianz, uno de los mayores tenedores privados de deuda española es de origen alemán, aunque la deuda está adquirida desde terceros países.

En cuanto a las ausencias, tal vez Rusia sea la más destacada. Se trata de un espacio estratégico en el mercado de deuda mundial, ya que gestiona una enorme inversión, ligada en buena medida a algunos fondos soberanos que alcanzan en 2009 los 168 millardos de dólares, el séptimo país en importancia a nivel mundial. Esto no significa necesariamente que fondos de origen ruso no hayan realizado inversiones en deuda española, pero es bastante significativa la inexistencia de sociedades financieras allí instaladas.

Desde el punto de vista de las sociedades de inversión, los datos analizados revelan que un total de 259 empresas han adquirido obligaciones emitidas por el Tesoro español por valor de 11.921 millones de dólares ${ }^{3}$. No obstante, a pesar del importante número que declaran este tipo de activos, existe una fuerte concentración del capital financiero en un reducido número de entidades. Las 11 mayores sociedades europeas absorben un 51,16\% del valor total de los títulos analizados, por lo que hay un nivel de concentración muy elevado de capital financiero en manos de un reducido número de empresas, que podrían en la práctica intervenir en el mercado de forma coordinada para modificar el interés de las emisiones. Algunas de ellas, como es el caso de BlackRock Investment Management, son filiales de sociedades de origen británico que forman parte de complejos empresariales deslocalizados por todo el mundo (Gráfico 2).

3 Se ha tomado como una sociedad diferente aquella que tiene un código de identificación propio. Puede ocurrir que existan sociedades con distinto código que pertenezcan al mismo grupo, el problema principal es que en la actualidad no hay información suficiente para poder establecer de forma rigurosa la estructura organizativa interna, por lo que puede ocurrir que sociedades de diferente identificador pertenezcan al mismo grupo financiero. 
Gráfico 2

DISTRIBUCIÓN DE LAS SOCIEDADES EUROPEAS DE INVERSIÓN. VALOR NOMINAL DECLARADO (AGOSTO DE 2012)

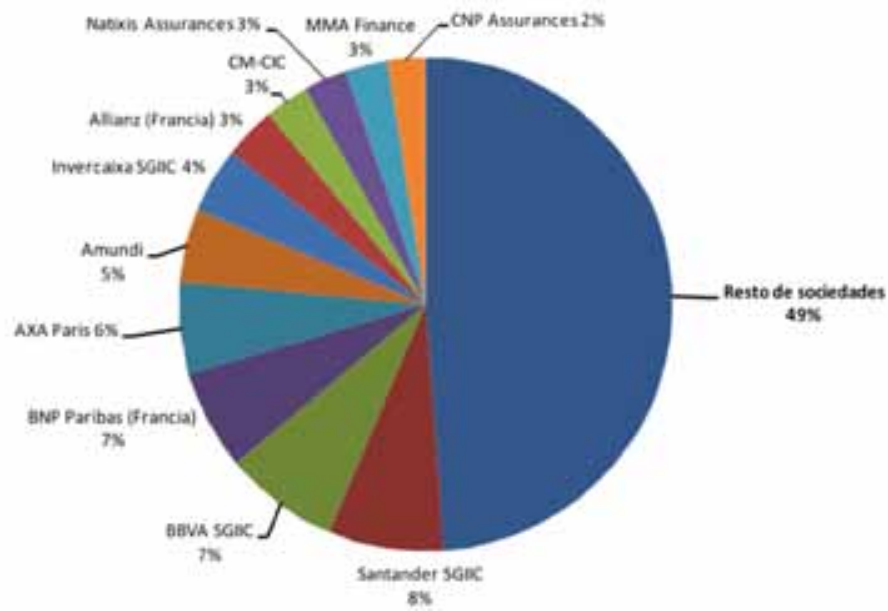

Fuente: Thomson Reuters al 14/08/2012 y elaboración propia.

Mapa 2

SOCIEDADES EUROPEAS QUE HAN ADQUIRIDO OBLIGACIONES. VALOR NOMINAL DECLARADO

(AGOSTO DE 2012, EN MILES DE US\$)

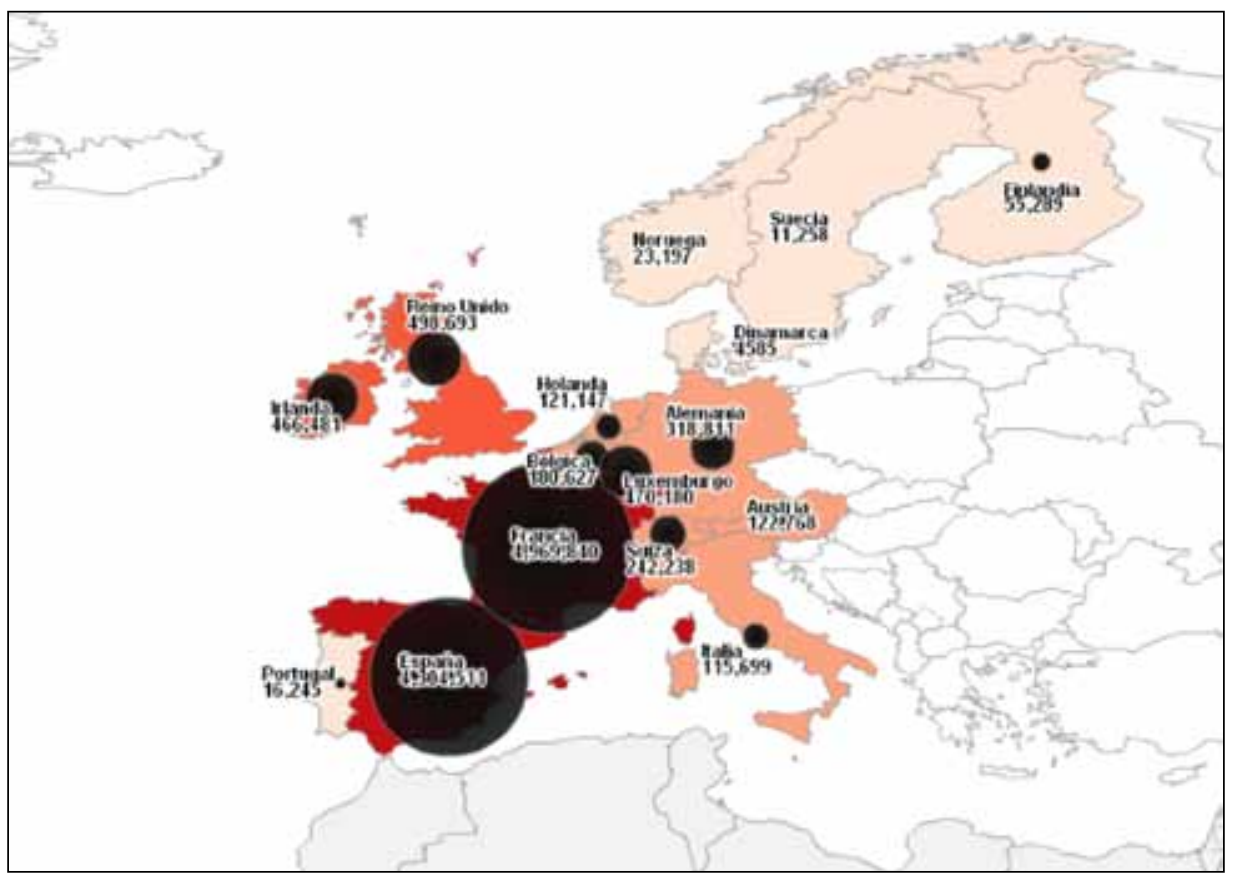

Fuente: Thomson Reuters al 14/08/2012 y elaboración propia. 
Mapa 3

SOCIEDADES EUROPEAS QUE INVIERTEN EN EL MERCADO ESPAÑOL (AGOSTO DE 2012)

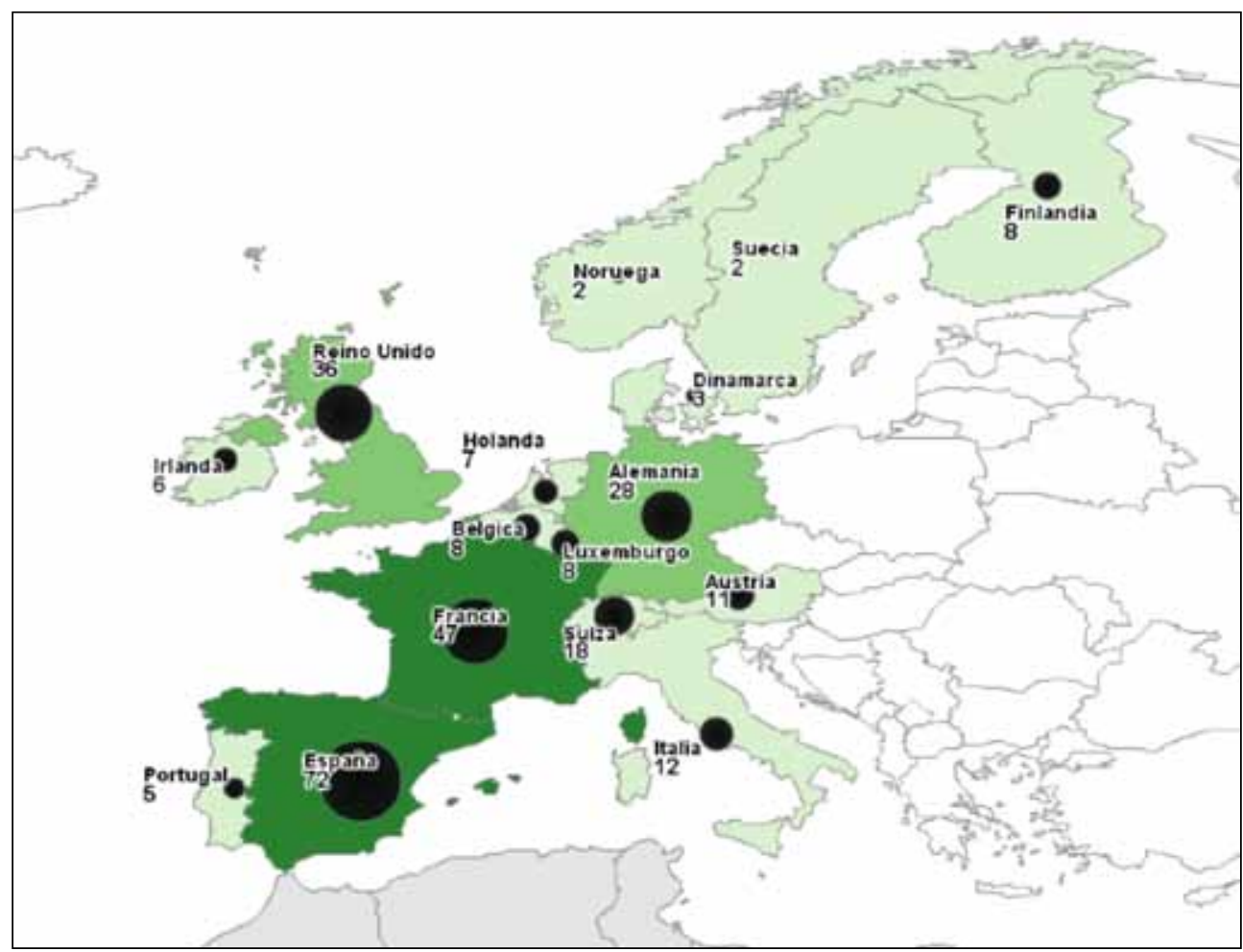

Fuente: Thomson Reuters al 14/08/2012 y elaboración propia.

\section{IV.2. Sociedades inversoras procedentes de América}

Fuera de Europa el número de inversores se reduce de forma importante. El bajo nivel de internacionalización del mercado español de deuda se pone de manifiesto en que solo dos países americanos, Estados Unidos y Canadá, tienen inversiones de obligaciones emitidas en 2012. Anteriormente sociedades radicadas en Bermudas habían adquirido este producto, aunque su presencia e impacto es meramente testimonial.

El comportamiento de las inversiones americanas es similar al que se realizan desde otros Estados. Si se analiza la distribución de los valores por fecha de emisión de la deuda se puede observar que las sociedades financieras han mantenido la proporción en el valor total de los activos. Entre 2006 y 2012 el valor ha ido oscilando de forma moderada en valores situados en torno al $2 \%$ del volumen total de la deuda agrupada por Estados.

La distribución de las inversiones realizadas está muy concentrada en torno a dos grandes sociedades financieras, The Vanguard Group Inc., y OppenheimerFunds Inc., que tienen obligaciones por valor de 120 millones de dólares y representan un 43,9\% del valor nominal de las obligaciones declaradas por sociedades de EE.UU. en 2012 (Tabla 3). 


\begin{tabular}{lrr}
\cline { 2 - 3 } & Valor par (en miles de US\$) & \% \\
\hline The Vanguard Group Inc. & 80.474 & 29,52 \\
OppenheimerFunds Inc. & 39.218 & 14,38 \\
State Street Global Advisors (SSgA) & 24.582 & 9,02 \\
Global Index Advisors Inc & 15.769 & 5,78 \\
Loomis, Sayles \& Company LP & 11.951 & 4,38 \\
Resto de sociedades & 100.647 & 39,62 \\
Total general & $\mathbf{2 7 2 . 6 4 1}$ & $\mathbf{1 0 0 , 0 0}$ \\
\hline
\end{tabular}

Fuente: Thomson Reuters al 14/08/2012 y elaboración propia.

Sin embargo el volumen más importante de inversión estadounidense no procede de sociedades localizadas en EE.UU. sino de sus filiales europeas, que funcionan como operadores en el mercado financiero español. La City de Londres es el mayor espacio de operaciones financieras estadounidense en España. Todas las grandes y conocidas firmas de inversión (Goldman Sachs o JP Morgan entre otros) operan desde Reino Unido, pero el volumen de inversión es claramente inferior al tamaño de la corporación y a la magnitud con la que invierte en otros mercados.

Parece ser que las sociedades norteamericanas, al igual que ocurre con las de Alemania, bien sea directamente o a través de filiales europeas operan en deuda española con valores muy por debajo de su capacidad económica real en el mercado financiero internacional.

\section{IV.3. Las inversiones procedentes de Asia y África. Una distribución desigual}

Una de las debilidades más significativas de la geografía financiera española es el escaso nivel de diversificación de las sociedades de inversión que adquieren su deuda. Resulta especialmente llamativa la ausencia de empresas financieras procedentes de espacios económicos emergentes, especialmente Rusia, India o Brasil. Como ya se ha visto anteriormente, la ausencia de tenedores de deuda registrados no significa necesariamente la inexistencia de inversiones de esos espacios, que pueden realizarse desde terceros países. Sin embargo, esta ausencia pone en evidencia el bajo nivel de relación que existe entre la economía española y unos espacios emergentes que en la actualidad representan una parte importante del crecimiento económico mundial.

Lo mismo ocurre con algunos Estados que cuentan con importantes fondos soberanos de inversiones. Junto al ya analizado caso de Rusia, habría que añadir algunas ausencias procedentes de Sudamérica, entre las que destaca el caso de Venezuela, o algunos países árabes. Todos estos espacios cuentan con fondos soberanos de alta liquidez obtenidos en gran parte por el incremento del precio del petróleo y el gas. Emiratos Árabes Unidos (Abu Dhabi Investment Authority), Noruega (Government Pension Fund-Global), Arabia Saudí (SAMA Foreign Holdings) y Kuwait (Kuwait Investment Authority) tienen los fondos soberanos más 
grandes del mundo y son potenciales inversores de deuda. En un segundo plano están los casos de Rusia (National Welfare Fund), Libia (Lybian Investment Authority) o Kazajistán (Kazakhstan Investment Authority) entre otros países con elevada capacidad financiera. Habría que valorar la posibilidad de diversificar este mercado geográfico, incorporando nuevos espacios que poseen en la actualidad una elevada capacidad de inversión exterior.

Tabla 4

PRINCIPALES TENEDORES ASIÁTICOS DE OBLIGACIONES (2012)

\begin{tabular}{lrr}
\cline { 2 - 3 } & Valor par (en miles de US\$) & $\mathbf{\%}$ \\
\hline Mitsubishi UFJ Asset Management Co Ltd & 86.377 & 33,83 \\
Nomura Asset Management Co Ltd & 38.743 & 15,18 \\
Nikko Asset Management Co Ltd & 35.954 & 14,08 \\
Daiwa Asset Management Co Ltd & 12.490 & 4,89 \\
STB Asset Management Co Ltd & 11.398 & 4,46 \\
BNP Paribas Investment Partners Japan Ltd & 10.039 & 3,93 \\
Resto de sociedades & 60.293 & 23,63 \\
Total general & $\mathbf{2 5 5 . 2 9 4}$ & $\mathbf{1 0 0 , 0 0}$ \\
\hline
\end{tabular}

Fuente: Thomson Reuters al 14/08/2012 y elaboración propia.

¿Significa esto que los países emergentes no invierten en deuda española? No se puede afirmar en absoluto en la medida en que, como ya hemos dicho anteriormente, pueden operar a través de sociedades localizadas en terceros países, una información que habría resultado muy valiosa para este trabajo pero que no se ha podido acceder.

Desde el punto de vista territorial desde Asia operan en España sociedades localizadas en Japón como país que capitaliza la inversión, ya que absorbe el 95,6\% del valor total de la deuda adquirida. Habría que añadir los casos de Hong Kong y Taiwán, que representan un $3,6 \%$ y un $0,8 \%$ respectivamente. Es probable que las sociedades localizadas en Hong Kong estén ligadas a capitales de procedencia china, un hecho que explicaría al menos parcialmente la elevada proporción de deuda adquirida en yuanes. El último operador de obligaciones procede de Singapur, aunque su participación en el valor global es testimonial.

Tal vez las incertidumbres financieras de la economía española están atrayendo algunos nuevos inversores de espacios donde tradicionalmente no se ha operado en el mercado español. Suráfrica es el único caso de inversión desde África; sin embargo el primer dato llamativo es que no tiene una tradición en este tipo de inversiones en España. La inversión se ha concentrado exclusivamente entre 2010 y 2012 aunque el volumen global de la misma es aún muy reducido. La incorporación de nuevos inversores procedentes de otros espacios emergentes como es el caso sudafricano es un hecho que en principio habría que valorar de manera positiva, en la medida en que el mercado financiero podría diversificar sus fuentes de financiación e integrar en su sistema capital procedente de nuevos espacios políticos, un dato relevante desde el punto de vista geopolítico. 
Desde el punto de vista de la estructura empresarial, la concentración financiera en pocas manos sigue siendo la tónica dominante en estos espacios, al igual que ocurre en Europa y América. El proceso de acumulación de capital es aún mayor, ya que un 76,37\% del valor nominal de la inversión procedente de Asia está en manos de cinco sociedades financieras, la mayor parte de ellas filiales de los grandes zaibatsu japoneses (Tabla 5).

\section{CONCLUSIONES}

La utilización de información financiera en el análisis espacial puede ser un instrumento valioso para ayudar a comprender las relaciones económicas y políticas entre distintos Estados. La evolución de la crisis financiera en el seno de la UE ha puesto de manifiesto la existencia de una correlación directa entre los flujos de inversiones financieras internacionales y las relaciones políticas que se dan entre sus Estados miembros. El manejo de esta información permite conocer con mayor detalle algunas de las consecuencias espaciales que ha generado este proceso.

El mercado de adquisición de deuda española se encuentra reducido a un grupo reducido de grandes sociedades, que controlan una parte importante del mismo. Los datos analizados ponen de manifiesto que una parte sustancial de las obligaciones declaradas en el mercado secundario están en posesión de un selecto grupo de empresas. Quizás se habla con una cierta precipitación del carácter anónimo de los mercados, ya que con la información adecuada es posible determinar quiénes son los responsables últimos de una determinada inversión y desde dónde se realiza la misma.

Las empresas financieras utilizan la localización espacial como herramienta estratégica en el proceso de toma de decisiones. Su estructura organizativa, altamente diversificada y con un importante nivel de especialización, considera su posición en el territorio como un elemento que juega un papel destacado. Esta estructura tiene un rango global y las inversiones en deuda se realizan de forma compleja, a través de diversas sociedades localizadas en los grandes centros financieros internacionales. Aunque es un tanto arriesgado hablar de cartelización del espacio financiero, la concentración de inversiones en un reducido número de empresas posibilita el desarrollo de este tipo de prácticas.

$\mathrm{Al}$ contrario de lo que ocurre en otros sectores de actividad económica, especialmente en la industria, donde las distintas filiales de las grandes multinacionales tienen un elevado nivel de especialización interna y una localización selectiva en el territorio, en el sector financiero la estructura organizativa interna de las distintas delegaciones no presenta diferencias sustanciales. Su estructura organizativa se encuentra ligada a la existencia de una lógica de localización adecuada a la morfología de este sector.

Las pautas de localización de estas multinacionales de la gestión del capital parecen tener una doble vertiente. En primer lugar figura la instalación de filiales en los grandes mercados financieros internacionales, que permite una mayor cercanía a los inversores y facilita una diversificación de las oportunidades de negocio a escala global. En segundo lugar resulta llamativa la proliferación de numerosas sedes en países que cuentan con una legislación fiscal favorable. Los datos analizados en el mercado español ponen de manifiesto que algunas operaciones se realizan desde este tipo de espacios. Sería de gran utilidad por estudiar con detenimiento los flujos internos de capital que se produce entre las distintas filiales de estos conglomerados. 
Durante los últimos años se ha incrementando el papel que juega en la deuda española algunas sociedades financieras. Las más importantes son Santander Asset Management, BBVA Asset Management, BNP Paribas Asset Management, AXA Investment Managers y Amundi. Tres de cada diez dólares declarados en obligaciones están en manos de estas sociedades. El 60,4\% del valor nominal de las obligaciones emitidas en 2012 pertenece a diecisiete sociedades, eso sin tener en cuenta que muchas de ellas podría tener filiales que puedan poseer otra parte importante de la deuda. Hay por tanto una cierta macrocefalia financiera en la adquisición de deuda española, que está concentrada en un selecto grupo de sociedades de inversión localizadas en Francia y España. El valor nominal de los títulos de deuda declarado por las sociedades financieras localizadas en Francia es superior a las que se encuentran en España (39,9\% y $34,5 \%$ respectivamente).

La toma de decisiones final en la adquisición de deuda se encuentra en algunos centros financieros internacionales, todo apunta a que la City de Londres juega un papel clave en la situación actual. Los flujos financieros exteriores de deuda pública proceden principalmente de Francia seguido a mucha distancia por Reino Unido (City), y dos Estados, Luxemburgo e Irlanda, con una ventajosa estructura fiscal. Resulta un tanto llamativo la baja participación de sociedades financieras localizadas en Alemania. La posición política francesa y alemana podría estar condicionada, al menos parcialmente, a la exposición de sus inversores en el mercado español. El nivel de internacionalización en España está menos diversificado de lo que a priori podría parecer. Este elemento podría considerarse clave en términos de estrategia política internacional, en la medida en que el Estado español presenta aún un nivel de relaciones económicas y políticas todavía insuficientes con algunos espacios geoeconómicos dinámicos.

Resulta significativo el escaso papel de los países emergentes en el mercado financiero español. No se ha detectado o son muy marginales las inversiones desde sociedades localizadas en algunos países inversores por excelencia, como es el caso de China, Rusia, India o Brasil. Tal vez sea conveniente que España refuerce su papel en otros espacios diferentes al europeo. España está volcada en la UE para afrontar esta crisis y quizás sea necesario diversificar los esfuerzos hacia nuevos mercados desde una perspectiva geopolítica más global.

\section{REFERENCIAS}

AGNEW, J. (2012): «Putting Politics into Economic Geography». The Wiley-Blackwell Companion to Economic Geography (Barnes, T. J., Peck J. y Sheppard E., coord.). Chichester. Ed. John Wiley \& Sons, Ltd.

ALESSANDRINI, P. FRATIANNI M. Y ZAZZARO, A. (2009): The Changing Geography of Banking and Finance. Nueva York. Springer Science+Business Media.

BANCO DE ESPAÑA (2012). El mercado de Deuda Pública 2011. Madrid. Banco de España. Disponible en http://www.bde.es

CAPELLE-BLANCARD, G. Y TADJEDDINE, Y. (2010): The impact of the 2007-10 crises on the geography of finance. París. Centre D’Etudes Prospectives et D'Informations Internationales. Disponible en http://www.cepii.fr/anglaisgraph/workpap/pdf/2010/ wp2010-16.pdf

CLARK, G.L. AND WOJCIK, D. (2007): The Geography of Finance: Corporate Governance in the Global Marketplace. Oxford. Oxford University Press. 
CROKER, C. (2002): Globalization and Insecurity in the Twenty-First Century: NATO and the management of Risk. The International Institute for Strategic Studies. Nueva York. Oxford University Press.

DE WIJK, R. (2012): «The geopolitical consequences of the $€$-crisis». Disponible en http:// www.europesworld.org/NewEnglish/Home_old/Article/tabid/191/ArticleType/ArticleView/ArticleID/21997/language/enUS/Thegeopoliticalconsequencesofthecrisis .aspx

DEL RÍO Y MARÍN (2010): «La ampliación de los diferenciales soberanos en la zona del euro durante la crisis». Boletín Económico del Banco de España. Noviembre de 2010, 82-92.

DEPETRIS, N. M. (2012): The New Geography of Capital Flows. Moscú. SKOLKOVO Institute for Emerging Market Studies.

DERUDDER, B., HOYLER, M. AND TAYLOR, P. (2011): «Goodbye Reykjavik: international banking centers and the global financial crisis». Área, $\mathrm{n}^{\circ} 43,173-182$.

DRUCKER, P. (1997) «The global economy and the nation-state». Foreign Affairs. Vol. $76, \mathrm{n}^{\circ} 5$.

DURDEN, T. (2012): «When It All Goes Bidless: What Losing \$1 Billion In 100 Milliseconds Looks Like». Zero Hedge. Disponible en http://www.zerohedge.com/news/when-itall-goes-bidless-what-losing-1-billion-100-milliseconds-looks

EICHENGREEN, B Y O'ROURKE, K (2010): «A tale of two depressions: What do the new data tell us?». Vox. Disponible en http://www.voxeu.org/article/tale-two-depressions-redux

ELLIOT, M. (2008): «A tale of three cities». Time. 17 de enero de 2008.

FRENCH, S.; LEYSHON, A. Y THRIFT, N. (2009): «A Very Geographical Crisis: the Making and Breaking of the 2007-2008 Financial Crisis». Cambridge Journal of Regions, Economy and Society $\mathrm{n}^{\circ} 2$ (2), 287-302.

GARCÍA-VAQUERO, V. Y CASADO, J.C. (2011): «El mercado español de deuda del Estado: desarrollos desde el inicio de la crisis». Boletín Económico del Banco de España. Septiembre de 2011, 89-98.

GOURINCHAS, P. REY, H. Y TRUEMPLER, K. (2011): «The Financial Crisis and The Geography of Wealth Transfers». Journal of International Economics n ${ }^{\circ} 88,266-283$.

HALL, S. (2012): «Geographies of money and finance II: Financialization and financial subjects». Progress in Human Geography, vol. 36 n 3 , 403-411.

HART, G. (2010): «Redrawing the map of the world? Reflections on the World Development Report 2009». En Economic Geography, vol. 86 n 4, 341-350.

HAU H. Y REY, H. (2008): «Global portfolio rebalancing under the microscope». En National Boureau of Economic Research. Working Paper Series $n^{\circ}$ 14.165. Cambridge. Disponible en http://www.nber.org/papers/w14165

ICEX (2010): Nota sobre los fondos soberanos chinos. Pekín. Oficina Económica y Comercial de la Embajada de España en Pekín.

INAYATULLAH, S. (2011): «Los futuros del mundo y la crisis financiera global: narrativas que definen y escenarios que crean». Ekonomiaz, n ${ }^{\circ}$ 76, 64-91.

LAURICELLA T. Y MC. KAY, P. (2010): «Dow Takes a Harrowing 1,010.14-Point Trip». The Wall Street Journal. 7 de mayo de 2010.

LEE, R., CLARK, G., POLLARD, J., Y LEYSHON, A. (2009): «The remit of financial geography before and after the crisis». Journal Economy Geography n 5, 723-747. 
LUTTWAK, E. N. (1995): «L〉arsenal de la géo-économie». Revue des deux mondes. Abril de 1995, 119-172.

MARINI G. Y PIERSANTI G. (2012): «Models of Speculative Attacks and Crashes in International Capital Markets». CEIS Tor Vergata Research Paper Series, vol. X, $\mathrm{n}^{\circ}$ 245.

MÉNDEZ, R. (2011): El nuevo mapa geopolítico del mundo. Madrid. Tirant lo Blanch.

MÖCKLI, D. (2012): Strategic Trends 2012. Key Developments in Global Affairs. Center for Security Studies. Zurich. Disponible en http://www.css.ethz.ch/publications/Strategic_Trends_EN

NANTO, D. K. (2009): «The Global Financial Crisis: Analysis and Policy Implications». CRS Report for Congress. Disponible en http://www.fas.org/sgp/crs/misc/RL34742.pdf

OAKLEY, D. (2012): «Investors taking huge sums out of eurozone bonds». En Financial Times. 17 de Abril de 2012. Disponible en http://www.espm.br/rjclipping/2012/ abril/43057.pdf

PUIG SOLER, S. (2011): «Los flujos financieros: amenaza estratégica en un mundo globalizado». Monografías del CESEDEN, $\mathrm{n}^{\circ}$ 124, 333-378.

SALAMA, P. (2012): «Preguntas y respuestas sobre la crisis mundial». Nueva Sociedad, $\mathrm{n}^{\circ}$ 237, 50-64.

SEO, B (2011): «Geographies of Finance : Centers, Flows, and Relations». Hitotsubashi Journal of Economics, $\mathrm{n}^{\circ}$ 52, 69-86.

THE Z/YEN GROUP (2012): The Global financial Centres Index 2011 . Disponible en http:// www.longfinance.net/Publications/GFCI\%2012.pdf

WALLERSTEIN, I. (2012): «Crisis estructural en el sistema-mundo. Dónde estamos y a dónde nos dirigimos». Monthly Review, vol LXII n ${ }^{\circ} 10$ marzo de 2011. Disponible en http://www.cienciasocialcritica.com/2/9

WÓJCIK, D. (2009): «Financial centre bias in primary equity markets». Cambridge Journal of Regions, Economy and Society, n ${ }^{\circ}$ 2, 193-209.

WÓJCIK, D. (2012): «The End of Investment Bank Capitalism? An Economic Geography of Financial Jobs and Power». Economic Geography, n $4,345-368$. 